\title{
PERCEPCIÓN DE COMPETITIVIDAD PERSONAL. UN ESTUDIO DESDE LA PERSPECTIVA DE LA FELICIDAD, EL BIENESTAR Y LA EDUCACIÓN EN EGRESADOS UNIVERSITARIOS
}

PERCEPTION OF PERSONAL COMPETITIVENESS. A STUDY FROM THE PERSPECTIVE OF HAPPINESS, WELL-BEING AND EDUCATION IN UNIVERSITY GRADUATES

Ahumada Tello, Eduardo (Universidad Autónoma de Baja California) *

\section{RESUMEN}

La competitividad es un tema de estudio que afecta a diferentes aspectos del individuo y de las organizaciones. La exigencia del ambiente moderno sobre la adquisición de habilidades y capacidades impulsa diversos estudios sobre la satisfacción que se logra al intentar cumplir con todos los requerimientos en el proceso de desarrollo. En esta investigación se busca encontrar una relación entre competitividad y las variables de felicidad, bienestar y educación para validar la existencia de influencia significativa entre ellas. Para estos efectos se utilizó un análisis de correlación y de ecuaciones estructurales tipo cuantitativo en un estudio de caso con una muestra de 106 personas egresados universitarios. Los hallazgos principales indican que la felicidad es una percepción que puede presentarse como independiente de la competitividad. En cambio, el bienestar y la educación mantienen una relación más significativa entre los encuestados.

Palabras claves: competitividad, felicidad, bienestar, educación, ecuaciones estructurales. JEL: I31, C3, L84.

\begin{abstract}
Competitiveness is a subject of study that affects different aspects of the individual and of the organizations. The demand that the modern environment towards the acquisition of skills, abilities and capacities impels diverse studies on the satisfaction that is obtained when trying to fulfill all the requirements in the process of development. In this research, we try to find a relation between competitiveness and the variables of happiness, welfare and education to validate the existence or significant influence among them. For these effects a correlation analysis and quantitative structural equations were used in a case study with a sample of 106 university graduates. The main findings indicate that happiness is a perception that can be presented as independent of competitiveness. In contrast, welfare and education have a more significant relationship among respondents.
\end{abstract}

Key words: competitiveness, happiness, well-being, education, structural equations. JEL: I31, C3, L84.

* Facultad de Contaduría y Administración. eahumada@uabc.edu.mx

Recibido: Diciembre de 2016. Aceptado: Febrero de 2017. 


\section{INTRODUCCIÓN}

La competitividad en el elemento humano ha sido objeto de un constante análisis dentro del mundo empresarial, académico y social. Bajo este constructo económico-administrativo se busca el éxito en las actividades y planes, logrando como resultado final un conjunto de las capacidades y aptitudes en la ejecución de alguna actividad o proceso. Una persona competitiva es aquella que pone en práctica conocimientos relacionados con su área de conocimiento, en situaciones normales y emergentes (Rey, 2014). Es importante mencionar que el desarrollo competitivo en las personas ha sido una variable del éxito para muchas organizaciones.

Considerar que la competitividad juega un papel importante dentro de diferentes contextos en los que el individuo se desenvuelve es parte fundamental para todo proceso educativo y formativo del capital humano en las organizaciones. Por ejemplo, en un contexto laboral la empresa tiende al mejoramiento continuo ante sus adversarios del mercado, por lo que el desarrollo competitivo que se da en sus integrantes puede influir de manera estratégica sobre la creación de alianzas que brinden estabilidad en el entorno interno y en el externo sobre las situaciones sociales y la forma de ganar ante la competencia (Chatenier, Verstegen, Biemans, Mulder y Omta, 2010). Esto se verá reflejado al cumplir los objetivos organizacionales, desarrollando con esto a un factor humano con alto nivel de capacidades, competencia, bienestar y altos niveles de felicidad o autosatisfacción sobre un modelo competitivo que abre camino al éxito de la organización.

A través de diversos estudios se ha hecho mención de cómo múltiples factores pueden afectar el desarrollo competitivo personal y profesional de los individuos. Estos están relacionados con los contextos que determinan si la persona es feliz en su área de desarrollo o con las actividades que realiza, lo que infiere que es mediante el cumplimiento adecuado de estas acciones que se alcanza un mayor progreso (Paschoal, Torres y Barreiros, 2010). Así mismo se ha estudiado la influencia que se presenta en el bienestar y la forma que este es percibido como parte consecuente del desarrollo de competitividad, asumiendo que al elevarse el individuo es capaz de asumir responsabilidades en diferentes ambientes y con ello mejorar las relaciones interpersonales positivas (Moreno y Marrero, 2015).

En estos ámbitos se ha identificado que las aptitudes y capacidades se reflejan sobre la competitividad personal en el entorno. Estos pueden ser influenciados por la educación fundamentada en el conocimiento en los procesos para tomar decisiones y sobre el juicio del éxito de los demás (Olson, 2012). Es factible considerar los procesos educativos como uno de los factores del desarrollo en el mundo competitivo, es a través de estos que se trasmiten elementos intangibles tales como el conocimiento y la percepción de éxito a los estudiantes y demás agentes involucrados, lo que da lugar a aptitudes positivas sobre la faceta personal y profesional en el individuo (Johansen, 2014). A través del conocimiento adquirido, los individuos pueden tener un acceso directo a empresas donde se les permita poner en práctica tanto sus conocimientos como sus aptitudes, así como medir sus capacidades y determinar su desempeño en un entorno real, aprendiendo de las relaciones activas que se dan dentro de los organismos productivos (Ruiz, 2014).

El desarrollo competitivo que se da en las instituciones y organismos favorece a los individuos en el campo creativo haciendo dinámica su forma de relacionarse (Strand, 2011). Este fenómeno genera entornos adecuados de trabajo que influyen en la competitividad económica, comercial, académica y profesional de los empleados y trabajadores. Es por ello que la organización requiere fomentar un espíritu emprendedor entre sus integrantes como medio de desarrollo competitivo personal que al final también influirá en la competitividad 
organizacional (Mahdavi Mazdeh, Razavi, Hesamamiri, Zahedi y Elahi, 2013). De esta manera, se observa la importancia de fomentar las competitividad personal a través de actividades flexibles entre los individuos, así como el desarrollo del trabajo en equipo dentro de los procesos organizacionales que incidan en la creación de profesionales capacitados (Mantilla y García, 2010).

\section{PLANTEAMIENTO DEL PROBLEMA}

La importancia del desarrollo competitivo de las personas no ha sido un concepto que sobresalga en los temas de estudio, puesto que se ha considerado como una variable en el desarrollo empresarial y dentro del mercado organizacional. Esto es lo que ha generado repercusiones dentro de estos mismos ámbitos, así como la influencia que este tiene sobre otros ambientes que favorecen el crecimiento personal del individuo, considerando que la competitividad ha generado mayores y ambiciosos resultados en todos los campos.

Como se puede identificar, la competitividad se da en diferentes contextos de la vida del individuo como pueden ser en lo personal, académico, social, laboral, etc., el desarrollo adecuado de este se puede ver influenciado por diferentes factores que se relacionan con las capacidades y habilidades tanto personales como profesionales, entre estos factores se considera a la felicidad que adquiere un papel importante en el logro de los objetivos, como es el desarrollo activo de lo que se quiere, buscado una meta personal, también se consideran en este como las relaciones pueden aportar a este concepto dentro del ambiente propiciando el ser feliz.

El estado de bienestar puede verse reflejado de manera subjetiva por los individuos en los diferentes ambientes en los que se expone, basado de aprendizajes, experiencias prácticas y conocimientos adquiridos lo que sustenta un nivel competente favorable. Y la educación asumiendo la responsabilidad de potencializar a los individuos como medio de adiestramiento formándolos para asumir responsabilidades tomando actitudes, de este modo evidenciar las capacidades y habilidades del individuo en el campo laboral.

Se puede decir que la consideración más importante para alcanzar el éxito está en conocer la determinación competitiva personal del individuo al incursionarse a un nuevo entorno donde se enfrentará a nuevos retos, personas, objetivos, cambios, procesos, visiones, etc. Es por ello necesario conocer el nivel de desarrollo que tiene el individuo como estudiante (en sus diferentes niveles académicos) o trabajador (en sus diferentes roles laborales).

A esto se debe que hoy en día las empresas se han enfocado a ser cada vez más competentes ante el entorno, pero no han considerado al capital humano como clave del éxito competitivo, sin embargo, muchas instituciones y empresas no conocen estos factores que pueden ser importantes en la aplicación de sus miembros para garantizar resultados más evidentes en el ambiente competitivo. Esta investigación indaga sobre la relación entre la competitividad, la felicidad, el bienestar y la educación, todo ello desde un enfoque determinado por la percepción del individuo que se convierte en el elemento clave de este trabajo.

\section{Preguntas de investigación}

1. ¿Es la felicidad un factor que influye en la competitividad personal?

2. ¿Es el bienestar un factor que influye en la competitividad personal?

3. ¿Es la educación un factor que influye en la competitividad personal? 


\section{Hipótesis general}

H La felicidad, el bienestar y la educación influyen en la competitividad personal.

HO La felicidad, el bienestar y la educación NO influyen en la competitividad personal.

Hipótesis de investigación e hipótesis nulas

$\mathrm{H}_{1} \quad$ La felicidad es un factor que influye en la competitividad personal.

$\mathrm{H}_{1} 0$ La felicidad NO es un factor que influye en la competitividad personal.

$\mathrm{H}_{2} \quad$ El bienestar es un factor que influye en la competitividad personal.

$\mathrm{H}_{2} \mathrm{O}$ El bienestar $\mathrm{NO}$ es un factor que influye en la competitividad personal.

$\mathrm{H}_{3} \quad$ La educación es un factor que influye en la competitividad personal.

$\mathrm{H}_{3} \mathrm{O}$ La educación es $\mathrm{NO}$ es un factor que influye en la competitividad personal Objetivo general:

1. Determinar si la felicidad, el bienestar y la educación son factores que influyen en el desarrollo de la competitividad personal.

Objetivos específicos:

1. Determinar si la felicidad influye en la competitividad personal.

2. Determinar si el sentido de bienestar influye en la competitividad personal.

3. Determinar si la educación influye en la competitividad personal.

\section{Justificación de la investigación}

El desarrollo competitivo es un medio de crecimiento que brinda beneficios personales y profesionales en los diferentes ambientes en que el individuo se desenvuelve. La adquisición de nuevas capacidades, experiencias, conocimiento, destrezas, aptitudes y compromisos, así como asumir nuevos retos, metas, objetivos y estatus es lo que mueve a los individuos a desarrollarse cada vez más, a ser mejores y a proyectarse progresivamente en los cambios del entorno, esto es lo que busca un ser competente.

La proyección preponderante de esta investigación está en la determinación de que las grandes organizaciones que fungen como ente de desarrollo y crecimiento para los individuos, consideren los aspectos que son importantes en función a los buenos resultados, y el beneficio que se puede alcanzar con el paso del tiempo asumiendo un papel eficaz en la toma de decisiones, considerando al capital humano en todas sus dimensiones para el logro y alcance de éxito.

Desde un punto empresarial, la generación de nuevas estrategias en el mundo competitivo ha hecho que las organizaciones tomen en cuenta factores que contribuyan al logro de nuevos objetivos, si bien, esta investigación busca exponer a las empresas factores que puedan hacer accesible el desarrollo de las competencias que se dan entre sus miembros. A través de este se les proporcionará una base fundamental de la importancia que se debe considerar sobre los empleados y sus relaciones en el área de trabajo como el de los logros adquiridos personal y profesionalmente, para el compromiso ante el éxito con la empresa.

Desde muchos contextos el éxito ha sido un objetivo por alcanzar por los individuos, es importante hacer mención que el éxito es una escala de procesos y niveles propuestos para una meta, si el individuo es competente es su área de desarrollo y cumple con todas sus actividades, como resultado se obtendrá el éxito, pero el éxito no solo se basa en el alcance de 
un objetivo, sino, sobre la suma de estos, que beneficien activamente el desarrollo de las capacidades, como el desenvolvimiento de las habilidades que generan un resultado favorable. Dentro de esto se hacen evidentes las relaciones que se presentan por otros individuos y tienen una repercusión positiva o negativa, haciendo notar la felicidad del individuo que influyen en el bienestar que generan un nivel de aceptación del entorno para ejercerse como competidores en su entorno.

El problema de los grandes organismos yace en la influencia de transmitir felicidad a los individuos y la carencia de generar un bienestar en el ambiente, y la falta de responsabilidad de considerar a la educación con simiente de desarrollo competitivo. Esto se ve reflejado ante el problema que se presenta en las organizaciones, el inconveniente de las empresas no es ser altamente competitivos ante el mercado, este radica en mantenerse competente ante ellos, pero, no se alcanza el máximo aprovechamiento, ya que el personal no cubre este requerimiento exigido, sin embargo, algunas empresas no consideran los factores que están en juego para actuar sobre ellos. Esta investigación busca solucionar el problema identificando las variables que pueden mantener a los empleados al margen del desarrollo competente del mercado.

Con este estudio se podrá determinar la influencia que aporta la felicidad, el bienestar y la educación sobre la competitividad personal, esto para mejorar las condiciones que se presentan en los medios que el individuo se desenvuelve personal y profesionalmente. Para que favorezcan y den un resultado efectivo en las empresas competentes, cubriendo las necesidades del ambiente, así como la formulación de principios básicos que se ejercen en las grandes industrias.

Esto puede dar cabida a nuevos estudios en diferentes contextos y nuevas variables con las que se quiera tener distintos resultados ante las relaciones empresariales en sus diferentes áreas y sectores, enriqueciendo el conocimiento a través de los diferentes enfoques de investigación.

\section{REVISIÓN DE LITERATURA}

\subsection{La competitividad como modelo de éxito personal y profesional}

El estudio significativo de las competitividad personal y/o profesional se desarrolla en la aplicación de las dimensiones de ser competente en tres esferas distintas: 1) situación deseada, 2) proceso de desarrollo, 3) contexto de formación, que se respaldan en el desarrollo competitivo (Climént, 2012). Estas dimensiones impulsan al individuo a valerse de sus capacidades y habilidades, como la adaptación y creación de modelos para la resolución de conflictos a los que se ve expuesto. Es adecuado considerar que la competitividad forma parte de los individuos de manera innata y la idea es desarrollar esta capacidad al interactuar entre sí, de manera individual y grupal y en todo momento considerando el entorno donde se desenvuelve. Esta visión integral incrementa el estímulo necesario para considerar habilidades, conocimientos y aptitudes adecuadas en diferentes contextos sociales (Climént, 2014).

\subsection{Competitividad personal y/o profesional en su entorno}

El contexto organizativo busca alcanzar resultados que trascienden en los procesos con los que se puedan mejorar las estrategias internas y externas. La organización ha identificado al personal como factor de éxito y a los adversarios en el ámbito empresarial como medio de 
oportunidad de mercado. El campo competitivo donde las empresas son capaces de crear situaciones de ganar-ganar; mostrar astucia social; entendiendo las situaciones sociales; socializar, desarrollar, mantener y utilizar redes eficaces para interpretar situaciones a través de la escucha activa en todos los contextos en el que se ve involucrado en la consecución de sus metas y objetivos (Chatenier, Verstegen, Biemans, Mulder y Omta, 2010), dan un resultado progresivo en todas las áreas de trabajo, estimulando una cultura adecuada para que los miembros de la organización desarrollen sus capacidades y habilidades permitiéndoles alcanzar objetivos tanto personales como laborales en beneficio mutuo.

Un incremento en la percepción de competencia se da principalmente en las personas y en sus relaciones, pues es en ellas que se desarrollan para cumplir alguna visión o meta, compartiendo experiencias que favorecen el desempeño individual y abriendo camino para la eficiencia de los procesos grupales. La evidencia del desarrollo de competencias o desarrollo competente, destaca la importancia del compromiso para el cambio y para los ajustes en los procesos que sea necesario adecuar para elevar el nivel de percepción en el grupo de trabajo. La actitud individual afecta de manera directa los procesos de cambio y es en ellos que se hacen evidentes las capacidades de los individuos (Mantilla y García, 2010).

Es entonces que las empresas tienden a cambiar los perfiles profesionales a perfiles más competentes que puedan cubrir las demandas y necesidades que se dan en los diferentes sectores de la organización. A su vez, se han desarrollado estrategias directivas para la gestión por competencias (López, 2010), buscando con ello, un comportamiento que atienda los cambios que se presentan en un entorno altamente competitivo para intentar garantizar el éxito.

En muchos entornos se busca tener al personal adecuado que pueda manifestar una actitud competente y pueda desarrollar sus habilidades en las diferentes situaciones que se puedan presentar, proporcionando una mayor capacidad y beneficio en otros sistemas sociales. El ser competente radica en la aplicación de todos los conocimientos, actitudes como aptitudes, prácticas y procesos sobre el área o rama que se ha desarrollado, atendiendo siempre las singularidades y problemas que se presentan, de manera eficiente y estos son medios de movilidad ante las situaciones, poniendo en práctica una respuesta útil que garantiza elevar un ambiente favorable en la condición que se presenta (Rey, 2014).

A través de esto se puede identificar la necesidad de la competitividad, en el mundo empresarial se da en los altos niveles jerárquicos, ante las contingencias que se presentan en el entorno del mercado dando lugar a la aplicación de motivación al personal que dan una respuesta competitiva ante las situaciones del entorno, incrementando la capacidad de alcanzar una maximización de los beneficios mutuos garantizando los objetivos (La Madriz y Parra, 2016). Existen las competencias personales que se presentan en situaciones complejas, dando forma de resolución de problemas con una buena comunicación efectiva en el trabajo de grupo; así como las competencias profesionales que se generan en la administración de recursos, planificación, organización de estrategias y la exposición de resultados como la del proceso de información y datos (La Madriz y Parra, 2016). Estos actúan en las organizaciones como ventaja competitiva de manera interna ante otras empresas en el entorno económico, comercial y productivo.

Con esto se argumenta que los modelos competitivos se dan en los diferentes contextos en el que el individuo se desarrolle, en el trabajo, la escuela, grupos sociales, etc. Estimulando el desempeño y la forma de desarrollo de las actividades, es evidente que las buenas actitudes de conducta están presentes en los resultados, que repercuten de manera positiva o negativa en la comunicación activa de los individuos. Los resultados se pueden medir a través de la 
actitud de los miembros, el equipo de trabajo, así mismo conocer la comodidad que el empleado tiene en su área, el grado de felicidad que este tiene, y con ello poder definir la productividad competente que se da en cada uno de ellos, debido a que se considera que las conductas que se dan pueden transmitir estímulos de felicidad y de bienestar que modelan las relaciones en el trabajo (Essounga-Njan, 2014).

\subsection{Competitividad y felicidad}

Es importante considerar que el individuo se siente feliz en lo que hace y como lo hace en los diferentes medios de su desarrollo, puesto que este es un estímulo para un buen desempeño en sus actividades promoviendo ante el entorno una competitividad inherente. Dentro de estos aspectos la felicidad personal no solo se puede relacionar con el objetivo, sino también en el entorno o ambiente en el que este se desenvuelva, lo que son las relaciones personales, sociales, académicas, laborales y profesionales, por lo que las buenas actitudes de conducta se ven reflejadas en el trato directo que se pueden dar de manera positiva o negativa transmitidos en la comunicación activa de los individuos, proyectando un grado de felicidad transmitido que brinda reacciones que favorecen o desfavorecen el comportamiento ante el logro de los objetivos (Swaroop, 2016).

La felicidad en el individuo puede ir en aumento o en descenso, esto depende de muchos factores personales, familiares, amistades, religiosos, laborales, sociales, etc. Basado en las experiencias que este va teniendo y el desarrollo que va adquiriendo. Se puede hacer evidente que la felicidad en una etapa joven donde los individuos entran en un ambiente universitario, está en constante cambio debido a diferentes factores en el entorno (Posada y Aguilar, 2012). Dentro del ámbito laboral las relaciones internas que se dan en el trabajo tienen un soporte evidente que tratan con la felicidad del individuo como empleado, transmitidos en el respaldo de las actividades, la cooperación entre los miembros, así como el reconocimiento que se genera por parte de los directivos (Paschoal, Torres y Barreiros, 2010), esto es lo que trasciende basado en las competencias internas para el alcance de logros personales dentro de la organización, haciendo que el individuo se sienta feliz con lo que hace y en su área de trabajo.

Es necesario considerar que entre los aspectos que el individuo considera importantes, está el ser un elemento importante en su entorno laboral. Esta expectativa impulsa la sensación de felicidad ante la seguridad de ser parte de un grupo como miembro activo, garantizando su compromiso en el desarrollo de sus funciones, independientemente del entorno, asumiendo un papel relevante en el trabajo, la escuela, la sociedad, la iglesia, etc., lo que estimula el cumplimiento de las actividades y políticas que se han establecido (Field y Buitendach, 2011).

Con esto se puede mencionar que en un enfoque laboral la empresa busca un cambio hacia la satisfacción de los empleados, logrando con ello una estrategia que pueda integrar a la felicidad como una dimensión de compromiso laboral, pero se ha demostrado que no se puede tener una relación directa entre felicidad y productividad, a menos que estos estén enlazados con la organización (Álvarez y Muñiz, 2013). Se puede argumentar que el individuo es felizmente activo en su organismo productivo teniendo donde desarrollar sus capacidades y practicar la competitividad ante otros, mejorando las relaciones, estableciendo autonomía en la práctica.

Sin embargo, las personas tienen la capacidad de adaptarse a nuevas relaciones, trabajos, cambios económicos que se presenten, de tal modo que los cambios positivos como recompensa para la felicidad individual a través de logros y éxitos, encontrando situaciones 
que promuevan un comportamiento competitivo ante el ambiente para ser felices en el sentido de la vida laboral y personal (Lyubomirsky, 2008).

\subsection{Competitividad y bienestar}

El concepto de bienestar humano se debe considerar en el diseño de iniciativas en los diferentes campos en los que el individuo interactúa, en lo personal, académico, laboral, social, religioso, etc. Promoviendo la satisfacción en las oportunidades de aprendizaje, experiencias, crecimiento, la salud y el trabajo (Straume y Vittersø, 2015), de esta manera se puede tener un estímulo de desarrollo competitivo en las personas.

La incitación competente puede depender de las relaciones que se dan en el ambiente de desarrollo y como propuesta de alcance de nuevas metas, las condiciones donde se desarrollan las actividades influyen en el estímulo que el individuo puede percibir para llevar a cabo los procesos determinantes de un objetivo. Un ambiente positivo puede aumentar la confianza y seguridad de las personad atrayendo un estado de bienestar que les permita tener la libertad de participar activamente en el contexto competitivo demostrando sus habilidades (Brief, 2014), de lo contrario si el ambiente es inadecuado para los individuos, no encontrarán un estado de bienestar, desfavoreciendo la capacidad y el deseo de promoverse competentemente.

En un ámbito laboral, para garantizar el estado de bienestar de los trabajadores es importante comprender el desarrollo de las actividades sobre las capacidades de desempeño que pueden ejercer, puesto que la actividad física crea un desgaste que repercute al momento de atender las necesidades del área en el que se desarrolla y en las iteraciones que se presentan dentro de la misma. El estado de bienestar se puede desligar de las reacciones del cuerpo que se dan el proceso productivo, lo que puede influir de manera negativa reduciendo la capacidad cognitiva de la resolución de los problemas, y la manera productiva ante la detección pertinente de la sobrecarga de trabajo, apoyan la fluidez de respuesta ante las situaciones que se pueden presentar (Zak y Winn, 2016), por ello es importante tener en cuenta que el estado de bienestar puede ser un factor de competencia personal y profesional ante el desarrollo adecuado de las actividades del individuo en su actividad laboral.

Las organizaciones tienen que ejercer un proceso determinante para establecer medios competitivos internos que se puedan ver reflejados externamente ante sus adversarios, esto implica una clara resolución del estado de bienestar con sus empleados contribuyendo al crecimiento personal y profesional dentro de las áreas de trabajo estableciendo relaciones optimistas y de esta manera favorecer su estado subjetivo permitiendo desarrollarse con nuevas experiencias y la adquisición de nuevos conocimientos. Es así como en este estado psicológico es factible garantizar la aceptación de las condiciones que le permitan mejorar, así como progresar en las relaciones de manera positiva y pueda manejar el entorno (Moreno y Marrero, 2015), teniendo esto promovido, las competencias dentro de la organización serán más evidentes para maximizar el resultado deseado.

En estos contextos el bienestar comprende el fenómeno de la felicidad, así mismo, se han estudiado el optimismo disposicional y el bienestar subjetivo mediante diversas medidas: felicidad (Lyubomirsky, 2008), emociones positivas y emociones negativas (Álvarez y Muñiz, 2013) y satisfacción en los dominios vitales de estudios, pareja, salud y ocio (Achor, 2010), por lo que se han encontrado asociaciones entre género de satisfacción con el trabajo, los estudios, la satisfacción vital, la felicidad y el optimismo. Además de tener una relación sentimental se relacionan con la satisfacción en pareja y así como las condiciones laborales con satisfacción vital, a través de esto surge un rasgo de personalidad relevante para mejorar 
el bienestar subjetivo de los individuos (Marrero-Quevedo, Carballeira-Abella y GonzálezVillalobos, 2014).

Sin embargo, se pueden encontrar requerimientos que determinan la importancia de la felicidad y el estado del bienestar entre las personas, a través de los procesos sociales como forma del progreso económico desde el ámbito laboral (Castellanos-Cereceda, 2013), permitiendo ver el avance dentro de una sociedad que se refiere a la integración de aspectos sociales y personales, que tratan de influir a un entorno cada vez más competente.

\subsection{Competitividad y educación}

Un desenlace competitivo se puede ver reflejado bajo la orientación de las capacidades y habilidades del individuo en el área donde puedan desarrollarse de manera efectiva y eficazmente (Coetzee, Bergh y Schreuder, 2010), a través de esto pueden llegar a ejercer un desarrollo cada vez más competente ante los demás, provocando progresivamente la resolución de problemas que se presentan. Siendo que la educación estimula un ambiente creativo para el desenvolvimiento personal y profesional ante los diferentes campos competentes, favoreciendo al individuo a conocer sus destrezas como el desarrollo de los mismos para garantizar el éxito para un resultado futuro (Hudec, Susnea, Bihar y Kirinić, 2015).

Sin embargo, una competitividad justa no se da sin la responsabilidad de asumir los resultados del objetivo planteado, así como el proceso de juicio ante la interacción con otros grupos competentes (Olson, 2012), estos son adquiridos en la educación, dado sobre varias disciplinas, relacionando las actividades prácticas en las que los estudiantes pueden desarrollarse en sus contextos personales y profesionales. Haciendo evidente el desarrollo de los campos creativos en el contexto educativo, se menciona que a través de este se adquieren conocimientos y aptitudes que influyen en el desarrollo de las competencias y, por lo tanto, de la percepción de ser competitivo (Reynolds, Stevens y West, 2013).

Considerando los aspectos relevantes de la educación y las relaciones que se dan sobre los individuos se hace un énfasis en que este brinda conexiones que favorecen a los estudiantes a involucrarse en el ámbito laboral de manera directa (Ramírez y Cárdenas, 2013). $\mathrm{Y}$ el beneficio que se obtiene al poner en práctica los conocimientos y procedimientos adquiridos de manera activa en la resolución de casos reales (Ruiz, 2014), de esta manera provoca personalmente un semblante competente que da lugar a beneficios profesionales.

\begin{tabular}{|c|c|}
\hline \multicolumn{2}{|c|}{$\begin{array}{c}\text { CUADRO 1. AUTORES DEL ESTUDIO DE COMPETITIVIDAD, } \\
\text { FELICIDAD, BIENESTAR Y EDUCACIÓN }\end{array}$} \\
\hline Variables de estudio & \multicolumn{1}{c|}{ Autores relevantes } \\
\hline Competitividad & $\begin{array}{l}\text { (Chatenier, Verstegen, Biemans, Mulder y Omta, 2010), (Mantilla y García, 2010), (Rey, } \\
\text { 2014), (Climént, 2012), (La Madriz y Parra, 2016), (López, 2010), (Climént, 2014) }\end{array}$ \\
\hline Felicidad & $\begin{array}{l}\text { (Essounga-Njan, 2014), (Paschoal, Torres y Barreiros, 2010), (Field y Buitendach, 2011), } \\
\text { (Posada y Aguilar, 2012), (Lyubomirsky, 2008), (Álvarez y Muñiz, 2013). }\end{array}$ \\
\hline Bienestar & $\begin{array}{l}\text { (Brief, 2014), (Zak y Winn, 2016), (Moreno y Marrero, 2015), (Straume y Vittersø, 2015), } \\
\text { (Achor, 2010), (Marrero-Quevedo, Carballeira-Abella y González-Villalobos, 2014). }\end{array}$ \\
\hline Educación & $\begin{array}{l}\text { (Coetzee, Bergh y Schreuder, 2010), (Hudec, Susnea, Bihar y Kirinić, 2015), (Olson, 2012), } \\
\text { (Reynolds, Stevens y West, 2013), (Ramírez y Cárdenas, 2013), (Ruiz, 2014) }\end{array}$ \\
\hline Fuente: Elaboración propia. \\
\hline
\end{tabular}


Se han hecho estudios que hablan de las competencias y su aplicación en las organizaciones, en Argentina (Rey, 2014) se enfocó de la relación y la diferencia que existe entre la competencia personal y la competencia profesional que se da en las personas sobre sus contextos. Se encontraron estudios que presenta una relación de la felicidad en las áreas de desarrollo, en sus diferentes contextos en Brasil (Paschoal, Torres y Barreiros, 2010), que puede influir en el modelo competente influenciando una productividad efectiva.

A través de ello se presentan informes del estado de bienestar como el que se realizó en México (Moreno y Marrero, 2015), que refleja como la competitividad se da por el bienestar subjetivo y psicológico de las personas involucrando sus actividades y el resultado de las mismas. Y la percepción que tienen los individuos a determinar su vocación educativa (Coetzee, Bergh y Schreuder, 2010), que repercutirá a futuro en su desarrollo laboral de manera activa sobre un contexto competente.

\section{METODOLOGÍA DE LA INVESTIGACIÓN Y CUESTIONARIO}

El estudio se realizó a través del enfoque cuantitativo por medio de cuestionarios para determinar si existe una relación entre las variables, así como la recolección de datos para probar la hipótesis planteada. Esto se refleja en datos numéricos y estadísticos para establecer las relaciones existentes. Como investigación no experimental, con alcance correlacional se busca evaluar la relación que existe entre la variable dependiente e independiente y su comportamiento.

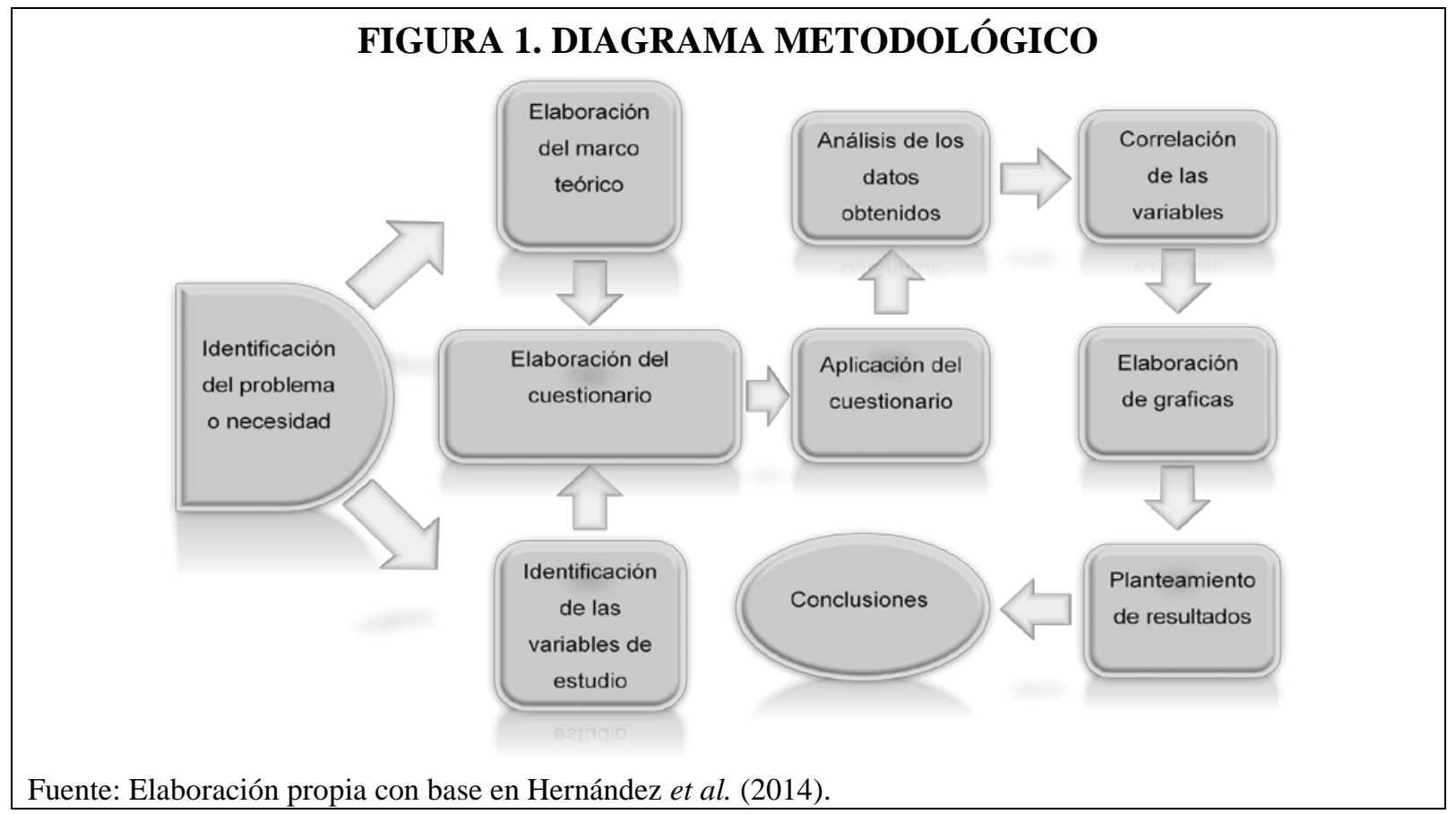

Se trata de una investigación aplicada, documental, correlacional, no experimental y transaccional; se busca con ello la comprensión del fenómeno de investigación, donde se realizó un análisis relativo a la influencia de tres variables sobre el constructo de competitividad.

Esta investigación se ha llevado a cabo con egresados de programas de estudio en Tijuana, México, de nivel superior para conocer el comportamiento competitivo personal 
determinado por las variables de felicidad, bienestar y educación. En el cuadro 2 se ilustran los datos principales del universo de estudio.

\begin{tabular}{|c|c|}
\hline \multicolumn{2}{|c|}{ CUADRO 2. UNIVERSO DE ESTUDIO } \\
\hline Concepto & Observación \\
\hline Marco muestral & Egresados universitarios de primer año \\
\hline Población & 106 individuos encuestados \\
\hline Estratos & Por tiempo de egreso \\
\hline
\end{tabular}

\section{RESULTADOS}

\subsection{Análisis de confiabilidad}

En el cuadro 3 se puede observar que, al realizar el análisis de confiabilidad de los resultados de la aplicación del cuestionario, se obtienen elevados índices de Alpha de Cronbach lo que indica que la confiabilidad del instrumento es significativa. También, se observa la relación entre factores y Alpha de Cronbach indica que el instrumento tiene validez de contenido significativo.

\begin{tabular}{|cccc}
\hline \multicolumn{4}{c|}{ CUADRO 3. ESTUDIO DE CONFIABILIDAD } \\
\hline Variable Dependiente & Variables Independientes & \# Ítems & Alpha de Cronbach \\
\hline Competitividad & & 9 & 0.852 \\
\hline & Felicidad & 2 & 0.876 \\
\cline { 2 - 4 } & Educación & 4 & 0.793 \\
\cline { 2 - 4 } & Bienestar & 3 & 0.778 \\
\hline Fuente: Elaboración propia. & & & \\
\hline
\end{tabular}

De acuerdo con Nunally (1967), los valores de Alpha de Cronbach entre 0.6 y 0.7, son aceptables para ítems que han de formar un mismo constructo en el área de las ciencias sociales. El resultado de 0.852 para competitividad, 0.876 para felicidad, 0.793 para educación y 0.778 para bienestar, indica una alta consistencia interna para cada variable.

\subsection{Correlación de Pearson}

Se puede observar en el cuadro 4 una alta correlación entre competitividad y felicidad ( $\mathrm{r}$ $=0.525 \mathrm{y} \mathrm{p}<0.001)$. De la misma manera la correlación entre competitividad y bienestar es representativa $(r=0.719$ y p $<0.001)$. Para finalmente identificar una alta correlación entre competitividad y educación $(\mathrm{r}=0.645$ y p $<0.001)$. Esta interpretación no implica causalidad, la relación solamente implica que las variables covarían entre sí. 


\begin{tabular}{|c|c|c|c|c|}
\hline \multicolumn{5}{|c|}{ CUADRO 4. CORRELACIÓN DE PEARSON } \\
\hline & & Felicidad & Bienestar & Educación \\
\hline \multirow{3}{*}{ Bienestar } & Correlación de Pearson & $0.680^{* *}$ & 1 & \\
\hline & Sig. (bilateral) & 0.000 & & \\
\hline & $\mathrm{N}$ & 106 & 106 & \\
\hline \multirow{3}{*}{ Educación } & Correlación de Pearson & $0.460 * *$ & $0.694 * *$ & 1 \\
\hline & Sig. (bilateral) & 0.000 & 0.000 & \\
\hline & $\mathrm{N}$ & 106 & 106 & 106 \\
\hline \multirow{3}{*}{ Competitividad } & Correlación de Pearson & $0.525 * *$ & $0.719 * *$ & $0.645 * *$ \\
\hline & Sig. (bilateral) & 0.000 & 0.000 & 0.000 \\
\hline & $\mathrm{N}$ & 106 & 106 & 106 \\
\hline
\end{tabular}

\subsection{Correlación de Spearman}

En el cuadro 5 se observa que el rango del coeficiente de Spearman obtenido entre las variables competitividad y felicidad (rho $=0.522, \mathrm{p}<0.001$ ), las variables competitividad y bienestar (rho $=0.691, \mathrm{p}<0.001$ ) y las variables competitividad y educación (rho $=0.650, \mathrm{p}$ $<0.001$ ), sustenta los resultados de la correlación de Pearson si existe relación entre las variables predictoras y la variable dependiente mayor a 0.500 y con un nivel de significancia menor a 0.01 .

\begin{tabular}{|c|c|c|c|c|}
\hline \multicolumn{5}{|c|}{ CUADRO 5. CORRELACIÓN DE SPEARMAN } \\
\hline & pearman & Felicidad & Bienestar & Educación \\
\hline \multirow[t]{3}{*}{ Bienestar } & Coeficiente de Correlación & $0.662^{* *}$ & 1 & \\
\hline & Sig. (bilateral) & 0.000 & & \\
\hline & $\mathrm{N}$ & 106 & 106 & \\
\hline \multirow[t]{3}{*}{ Educación } & Coeficiente de Correlación & $0.438^{* *}$ & $0.697^{* *}$ & 1 \\
\hline & Sig. (bilateral) & 0.000 & .0000 & \\
\hline & $\mathrm{N}$ & 106 & 106 & 106 \\
\hline \multirow[t]{3}{*}{ Competitividad } & Coeficiente de Correlación & $0.522 * *$ & $0.691^{* *}$ & $0.650 * *$ \\
\hline & Sig. (bilateral) & 0.000 & 0.000 & 0.000 \\
\hline & $\mathrm{N}$ & 106 & 106 & 106 \\
\hline
\end{tabular}




\subsection{Evaluación de hipótesis mediante estudio correlacional}

En el cuadro 6 se especifica que se rechazan las hipótesis nulas, el análisis de resultados indica que sí existe relación entre las variables independientes y la variable dependiente mayor a 0.500 y con un nivel de significancia menor a 0.01 .

\begin{tabular}{|ccc|}
\hline \multicolumn{3}{|c|}{ CUADRO 6. EVALUACIÓN DE HIPÓTESIS } \\
\hline Hipótesis & \multicolumn{1}{c|}{ Descripción } & \multicolumn{1}{c|}{ Conclusiones } \\
\hline $\mathrm{H}_{\mathbf{1}} \mathbf{0}$ & No hay relación entre competitividad y felicidad & No se acepta porque $\mathrm{p}<0.05$ \\
\hline $\mathrm{H}_{\mathbf{2}} \mathbf{0}$ & No hay relación entre competitividad y bienestar & No se acepta porque $\mathrm{p}<0.05$ \\
\hline $\mathrm{H}_{3} \mathbf{0}$ & No hay relación entre competitividad y educación & No se acepta porque $\mathrm{p}<0.05$ \\
\hline Fuente: Elaboración propia. & \\
\hline
\end{tabular}

En las correlaciones de Pearson y de Spearman se confirman los resultados de dependencia que se plantean en el estudio. La competitividad tiene relación con la felicidad, el bienestar y la educación. Estos hallazgos aseguran una tendencia hacia el fortalecimiento del capital humano que se integre a la planta de trabajo con una perspectiva humanista e integral.

\subsection{Ecuaciones estructurales}

Para realizar una validación adecuada de los supuestos planteados a partir de la teoría, se lleva a cabo el análisis de ecuaciones estructurales mediante el uso del software SPSS AMOS 21. Para ello se determina previamente que las respuestas a los cuestionarios se plantearan en escala de Likert con siete elementos que varían de percepciones de "Totalmente de acuerdo" a "Totalmente en desacuerdo". En la figura 2 se muestra el modelo de ecuaciones estructurales que describe la apreciación teórica de la relación de variables.

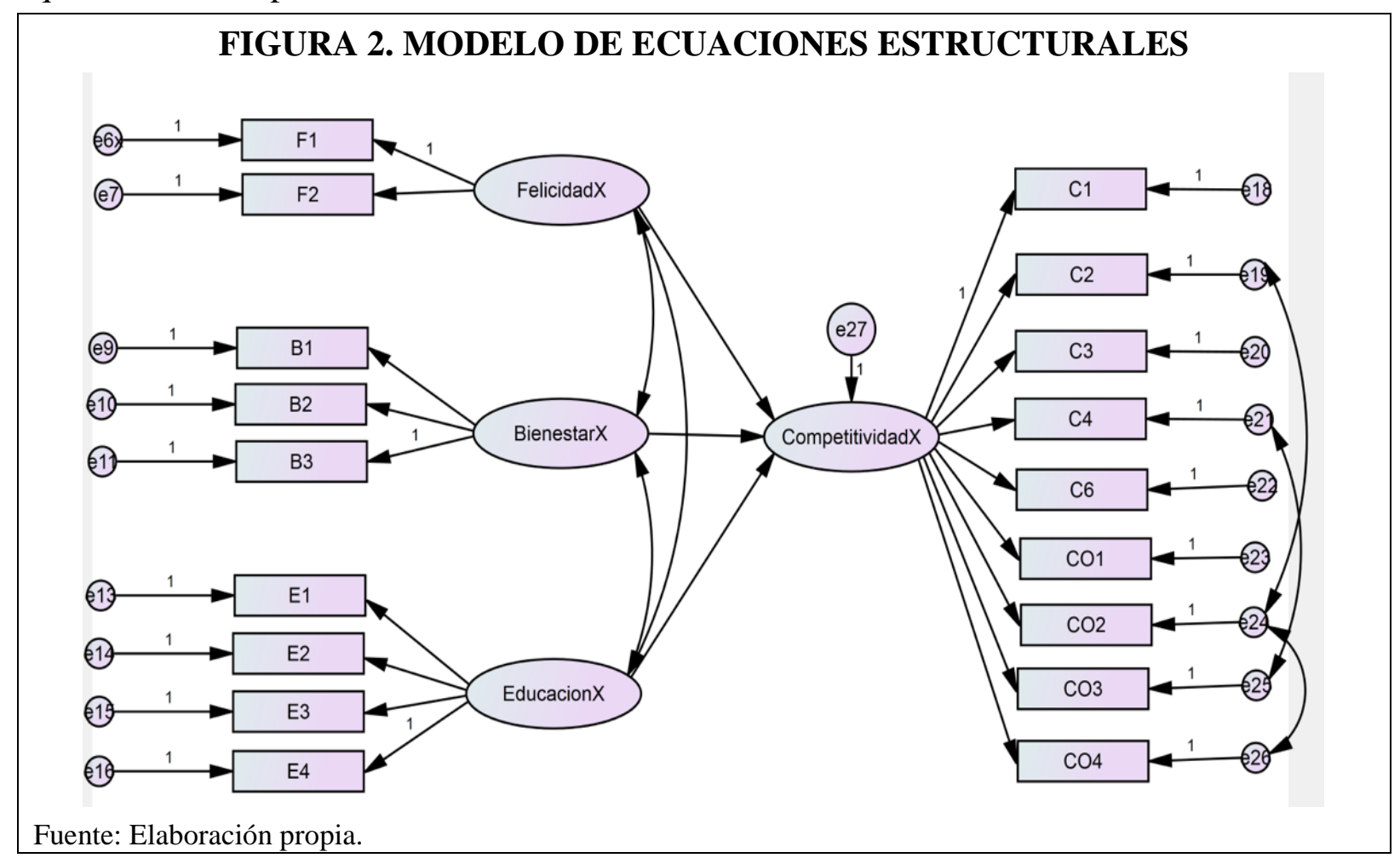


El modelo integra dieciocho variables observables, cuatro variables latentes, de las cuales tres son exógenas y una es endógena. En este sentido, dos variables observables definen el constructo y variable latente exógena de felicidad; tres variables observables definen el constructo y variable latente exógena de bienestar; cuatro variables observables definen el constructos y variable latente exógena de educación; y finalmente nueve variables observables definen el constructo y variable latente endógena competitividad.

En el cuadro 7 se observa la relación de covarianza entre las variables latentes endógenas del modelo, se observa un alto valor de covarianza y un elevado nivel de significancia.

\begin{tabular}{|c|c|c|c|c|c|c|c|}
\hline \multicolumn{8}{|c|}{ CUADRO 7. COVARIANZAS ENTRE VARIABLES LATENTES } \\
\hline & & & Estimación & S.E. & C.R. & $\mathbf{P}$ & Etiqueta \\
\hline FelicidadX & $<-->$ & Bienestar X & 0.754 & 0.165 & 4.585 & $* * *$ & par_18 \\
\hline BienestarX & $<-->$ & Educación X & 0.816 & 0.212 & 3.854 & $* * *$ & par_19 \\
\hline FelicidadX & $<-->$ & Educación X & 0.669 & 0.184 & 3.638 & $* * *$ & par_20 \\
\hline
\end{tabular}

En el cuadro 8 se observa los valores de regresión entre las variables acordes al modelo de la figura 2. En este sentido el valor de regresión de felicidad en relación a competitividad indica una alteración al modelo teórico. Este dato no concuerda con el planteamiento de una influencia entre estas dos variables latentes. En este escenario, el análisis estricto de ecuaciones estructurales manifiesta una diferencia con el análisis correlacional previo y complementa los resultados.

Así mismo, las estimaciones para el modelo de competitividad se encuentran en el cuadro 8. La estimación C.R. resulta en general significativa (>1.7) para la mayoría de las variables; excepto para competitividad<-felicidad con un valor de -0.797; el cual aporta un indicador de no significancia para el modelo. Las estimaciones con valores menores a 0.005 en el estadístico p de los elementos de estudio sugieren una alta significancia para esta variable que se indica, no así en la relación de variables latentes, donde se nuevo el valor de felicidad afirma que no existe significancia entre este constructo y la competitividad.

Cada variable observable indicada muestra una carga estimada representativa, salvo CO2 y CO3 que se mantienen por debajo de 0.6. De la misma manera, la variable latente Bienestar presenta una estimación menor a 0.6, lo que ilustra una significancia aceptable.

En el cuadro 9 se encuentran los valores más relevantes de la bondad de ajuste obtenido al realizar el análisis mediante la herramienta estadística seleccionada y que a continuación se ilustra y se describen los indicadores más relevantes.

En el cuadro 9 se observa que varios de los estadísticos recomendables validan el modelo propuesto para estudiar la relación entre las variables latentes de esta investigación. Se observa que el valor boostrap es superior al criterio de aceptación $(>0.05,0.574)$, de la misma manera, Chi Cuadrado $(>0.05,0.163)$, RMSEA $(<0.08,0.030)$ y PRATIO $(>-.5$, 0.874). Los estadísticos que no validan el modelo de manera óptima son GFI $(>0.90,0.808)$ y AGFI $(>0.90,0.760)$. Sin embargo, los resultados son suficientes para validar de manera aceptable el modelo teórico propuesto y ajustando las relaciones que se generaron entre las variables Competitividad<-Felicidad que no responden al modelo. 


\section{CUADRO 8. ESTIMACIÓN DE REGRESIÓN}

\begin{tabular}{|c|c|c|c|c|c|c|c|}
\hline & & & Estimación & S.E. & C.R. & $\mathbf{P}$ & Etiqueta \\
\hline CompetitividadX & $<---$ & FelicidadX & -0.110 & 0.138 & -0.797 & 0.425 & par_15 \\
\hline CompetitividadX & $<---$ & BienestarX & 0.425 & 0.209 & 2.034 & 0.042 & par_16 \\
\hline CompetitividadX & $<--$ & EducacionX & 0.647 & 0.231 & 2.793 & 0.005 & par_17 \\
\hline F1 & $<---$ & FelicidadX & 1.000 & & & & \\
\hline F2 & $<--$ & FelicidadX & 0.990 & 0.144 & 6.853 & $* * *$ & par_1 \\
\hline B1 & $<---$ & BienestarX & 0.840 & 0.132 & 6.351 & $* * *$ & par_2 \\
\hline B2 & $<---$ & BienestarX & 0.798 & 0.143 & 5.575 & $* * *$ & par_3 \\
\hline B3 & $<---$ & BienestarX & 1.000 & & & & \\
\hline E1 & $<--$ & EducacionX & 0.818 & 0.184 & 4.448 & $* * *$ & par_4 \\
\hline E2 & $<--$ & EducacionX & 0.911 & 0.227 & 4.008 & $* * *$ & par_5 \\
\hline E3 & $<--$ & EducacionX & 0.740 & 0.186 & 3.972 & $* * *$ & par_6 \\
\hline E4 & $<--$ & EducacionX & 1.000 & & & & \\
\hline $\mathrm{C1}$ & $<---$ & CompetitividadX & 1.000 & & & & \\
\hline $\mathrm{C} 2$ & $<--$ & CompetitividadX & 0.762 & 0.116 & 6.569 & $* * *$ & par_7 \\
\hline C3 & $<--$ & CompetitividadX & 0.830 & 0.156 & 5.309 & $* * *$ & par_8 \\
\hline $\mathrm{C4}$ & $<--$ & CompetitividadX & 0.892 & 0.160 & 5.573 & $* * *$ & par_9 \\
\hline C6 & $<--$ & CompetitividadX & 0.594 & 0.152 & 3.921 & $* * *$ & par_10 \\
\hline CO1 & $<---$ & CompetitividadX & 0.783 & 0.166 & 4.710 & $* * *$ & par_11 \\
\hline $\mathrm{CO} 2$ & $<---$ & CompetitividadX & 0.498 & 0.109 & 4.592 & $* * *$ & par_12 \\
\hline $\mathrm{CO} 3$ & $<--$ & CompetitividadX & 0.451 & 0.127 & 3.561 & $* * *$ & par_13 \\
\hline CO4 & $<---$ & CompetitividadX & 0.623 & 0.174 & 3.589 & $* * *$ & par_14 \\
\hline
\end{tabular}

Fuente: Elaboración propia.

\section{CUADRO 9. ESTADÍSTICOS DE BONDAD DE AJUSTE Y CRITERIOS DE REFERENCIA}

\begin{tabular}{|c|c|c|c|c|}
\hline & Estadístico & Abreviatura & Criterio & Resultados \\
\hline Boostrap & Bootstrap Method (n=106) & P Value & $>0.05$ & 0.574 \\
\hline \multirow{5}{*}{$\begin{array}{l}\text { Ajuste } \\
\text { absoluto }\end{array}$} & $\begin{array}{l}\text { Función de ajuste mínimo Chi } \\
\text { Cuadrado }\end{array}$ & $\chi^{2}$ & $>0.05$ & 0.163 \\
\hline & $\begin{array}{lll}\text { Función de ajuste mínimo Chi } \\
\text { Cuadrado / Grados Libertad (gl) }\end{array}$ & CMIN / gl & $<2$ & $221 / 202=\mathbf{1 . 0 9}$ \\
\hline & $\begin{array}{l}\text { Error de aproximación cuadrático } \\
\text { medio }\end{array}$ & RMSEA & $<0.08$ & 0.030 \\
\hline & Índice de bondad de ajuste & GFI & $>0.90$ & 0.808 \\
\hline & Índice ajustado de bondad de ajuste & AGFI & $>0.90$ & 0.760 \\
\hline $\begin{array}{c}\text { Ajuste } \\
\text { comparativo }\end{array}$ & $\begin{array}{l}\text { Índice esperado de validación } \\
\text { cruzado }\end{array}$ & ECVI & $\begin{array}{c}<\text { a modelo } \\
\text { saturado e } \\
\text { independencia }\end{array}$ & $\begin{array}{l}\text { Def: } \mathbf{3 . 0 8 3} \\
\text { Sat.: } \mathbf{4 . 8 1 9} \\
\text { Ind. } \mathbf{3 . 4 2 6} \\
\end{array}$ \\
\hline $\begin{array}{c}\text { Ajuste } \\
\text { Parsimonioso }\end{array}$ & Parsimonia & PRATIO & $>0.5$ & 0.874 \\
\hline $\begin{array}{c}\text { Tamaño de } \\
\text { muestra }\end{array}$ & N Crítico (CN) & $\begin{array}{l}\text { HOELTER } \\
0.05 \\
\end{array}$ & $\begin{array}{l}\text { Propuesto por } \\
\text { AMOS }\end{array}$ & 112 \\
\hline
\end{tabular}




\subsection{Evaluación de hipótesis en el estudio de ecuaciones estructurales}

Los resultados de la evaluación de hipótesis ilustrados en el cuadro 10, indican que dos de ellas son rechazadas (H20 y H30) y una es aceptada (H10). La que ha sido aceptada menciona que la felicidad no tiene relación con competitividad. Esto se fundamenta en base a los resultados de las estimaciones y del valor de significancia que se presenta en el estudio de ecuaciones estructurales. Las dos hipótesis corroboradas, confirman la evaluación de hipótesis mediante el análisis de correlaciones de Pearson y Spearman en el cuadro 4 y 5.

\section{CUADRO 10. EVALUACIÓN DE HIPÓTESIS CON MÉTODO DE REGRESIÓN}

\begin{tabular}{|c|c|c|}
\hline Hipótesis & Descripción & Conclusiones \\
\hline $\mathbf{H}_{1} \mathbf{0}$ & $\begin{array}{l}\text { No hay relación entre Competitividad y } \\
\text { Felicidad }\end{array}$ & $\begin{array}{l}\text { Se acepta porque Estimación }=-0.110 \text { y } \mathrm{P}= \\
0.425\end{array}$ \\
\hline $\mathbf{H}_{2} \mathbf{O}$ & $\begin{array}{l}\text { No hay relación entre Competitividad y } \\
\text { Bienestar }\end{array}$ & $\begin{array}{l}\text { No se acepta porque Estimación }=0.425 \text { y } \mathrm{P}= \\
0.042\end{array}$ \\
\hline $\mathbf{H}_{3} \mathbf{O}$ & $\begin{array}{l}\text { No hay relación entre Competitividad y } \\
\text { Educación }\end{array}$ & $\begin{array}{l}\text { No se acepta porque Estimación }=0.647 \text { y } \mathrm{P}= \\
0.005\end{array}$ \\
\hline
\end{tabular}

\section{DISCUSIÓN Y CONCLUSIONES}

Este estudio pone de manifiesto constructos que ponderan la capacidad de ser o percibirse como un ente competitivo a través de la evaluación de tres variables independientes relacionadas de manera teórica con competitividad: felicidad, bienestar y educación. Esta visión debe ser evaluada y ponderada para impulsar estudios futuros que corroboren los resultados obtenidos y mejoren las estrategias de aproximación al estudio, para que, al mismo tiempo, se estructuren instrumentos de medición más confiables y concisos.

En los resultados de la primera parte del estudio estadístico, los valores obtenidos confirman que existe una correlación significativa entre cada una de las variables del estudio. Se logra el rechazo de las hipótesis nulas y se valida que existe una correlación entre competitividad, felicidad, bienestar y educación. La diferencia ocurre cuando se lleva a cabo un estudio más estricto por medio del uso de ecuaciones estructurales. Esta aproximación provoca que se acepta una hipótesis nula y se establezca que no existe un sustento empírico suficientemente aceptable para validar los supuestos teóricos planteados.

Estos resultados parciales invitan a reflexionar sobre un aspecto relevante entre el concepto de felicidad y competitividad. Es común considerar que una persona que es competitiva es feliz, sin embargo, este estudio indica que no necesariamente ocurre así. Posiblemente, la lucha constante por mantener una ventaja competitiva en un ámbito laboral turbulento puede afectar la percepción de felicidad. El empleado puede presentar una alta capacidad competitiva pero no considerar que cumple sus expectativas de felicidad personal. Estos razonamientos aportan una perspectiva adicional al problema abordado y generan una nueva pregunta para futuras investigaciones sobre el tema: ¿es posible alinear los requerimientos competitivos con las expectativas de realización personal que incrementen la percepción de felicidad?

En todos los ámbitos el ser humano siempre ha buscado maneras de sobresalir, son sus capacidades y destrezas que lo hacen subsistir en el medio ambiente en el que se desarrolla, se ha envuelto en un contexto en donde los más capaces son los que predominan, es de este 
modo donde se dan las competencias personales en los individuos, demostrando sus habilidades y talentos adquiridos en su crecimiento, como la adaptación con el entorno. Estas competencias se hacen más evidentes en los diferentes contextos; en la escuela, el trabajo, la sociedad, los grupos sociales, el gobierno, etc., siempre en ventaja de otros individuos, desenvolviéndose de manera personal y profesionalmente.

Teniendo en cuenta este desarrollo, el ser humano tiende a buscar la felicidad en todos los ámbitos: en casa, en la escuela, con la pareja, en los regalos, en un paseo, en el trabajo, en los hijos, en el trabajo, etc. La felicidad es un estado emocional que se produce en la persona cuando cree haber alcanzado una meta deseada. Tal estado propicia paz interior, un enfoque del medio positivo, al mismo tiempo que estimula a conquistar nuevas metas.

Desde el punto de vista de esta investigación la información recabada sobre la felicidad y el bienestar se pueden considerar hasta cierto punto como sinónimos, en el sentido estricto de satisfacción que perciben las personas, favoreciendo el desarrollo competitivo de los individuos en sus medios. Sobre este mismo enfoque podemos ver que la educación juega un papel importante sobre este impulsando el crecimiento continuo sobre las situaciones que se presentan en las relaciones externas, como lo es en el campo laboral.

Sin embargo, es evidente que la necesidad competente de hoy tiende a asumir grandes cambios, responsabilidades, buenos o malos resultados, por ello las consideraciones sobre estos factores que actúan en conjunto con la competitividad personal, promuevan una actitud que mantenga a las personas felices que trasmitan un ambiente adecuado y se perciba un estado de bienestar para que el individuo pueda crecer y desarrollarse en su área activamente, buscando con ello una competitividad saludable en los procesos que se dan en los diferentes entes sociales. Esto con la finalidad de cumplir objetivos y estimular el mejoramiento del desempeño para que el logro organizacional sea el más óptimo.

En consideración al estudio teórico se puede inferir que las personas felices suelen ser más creativas, innovadoras, emprendedoras y con mayor tendencia a la interacción social y con actitud extrovertida, ocasionando la incitación competitiva ante al contexto. Por otro lado, se hace visible que el bienestar personal favorece las relaciones que se dan en el ambiente de manera subjetiva. Además, es factible mencionar que la educación funge como medio importante ante el desarrollo competitivo de los individuos, siendo generador de aptitudes competentes ante el medio, así como el adiestramiento para adoptar los cambios y obstáculos que se presentan en la vida y el medio laboral.

La educación adquirida por el individuo es un medio de transición hacia el campo empresarial o la incursión a los organismos productivos en los diferentes sectores sociales, sin embargo, hay empresas que presionan a su personal para el logro de los objetivos y esto ocasiona pérdidas económicas como de recursos humanos. Por ello, se recomienda considerar a la felicidad y el bienestar como factores que favorecen el estímulo competitivo del personal, a favor de un desarrollo educativo que propone a un individuo capaz en la resolución de problemas y conflictos, atrayendo resultados positivos y trascendentes en el desarrollo de las actividades organizacionales, como un paso al éxito empresarial.

A través de este podemos concluir que la competitividad personal es afectada por los factores de felicidad, bienestar y la educación. Así mismo hacen de este un proceso activo en las relaciones y actividades en las que el individuo se desenvuelve activamente. Estos factores pueden afectar de manera positiva o negativamente a la competitividad personal, esto depende de las influencias que recaigan en los desenvolvimientos progresivos del individuo o sobre el objeto de cubrir una necesidad, así como la de alcanzar un objetivo personal o profesional. 
Dando una respuesta estratégica de aplicación sobre el capital humano en todos los entornos, garantizando un desarrollo más activo y productivo, atendiendo la demanda competitiva que hoy en día se está dando en los ambientes laborales y evidenciando las capacidades del individuo como fuente eficaz de beneficios transcendentes hacia las organizaciones.

\subsection{Limitaciones del estudio}

Se estableció un límite geográfico para la aplicación del estudio en la ciudad de Tijuana, B.C. El grupo de estudio radicó sobre estudiantes y egresados de nivel superior. En la recolección de información teórica se consideran estudios con cinco años de referencia. Se han determinado las variables de estudio que fungen en el desarrollo o aplicación de la competitividad personal. El estudio se desarrolla como investigación cuantitativa. Los resultados cubren la finalidad del estudio planteado.

\subsection{Futuras líneas de investigación}

Para continuar con el proceso de validación de factores de percepción en el desarrollo de competitividad personal, es importante dar continuidad a este estudio. Se pretende confirmar los resultados en momentos futuros con elementos y muestras similares que ayuden a proponer modelos más ajustados al marco de referencia teórica sobre el constructo de competitividad personal, felicidad, estado del bienestar y educación. De la misma manera, se buscará aplicar estrategias de investigación cualitativa como lo son las entrevistas a profundidad, historias de vida, observación, etnografía y focus group. Esto con la finalidad de complementar la validez de los resultados al incluir conceptos de percepción.

Así mismo, la relación que se puede determinar entre factores de percepción como felicidad y estado del bienestar con la competitividad, se convierte en una línea de investigación adecuada para mejorar el funcionamiento de las organizaciones empresariales. De acuerdo a los primeros estudios indicados en este documento, el elemento o factor humano que se encuentra realizado como individuo o al menos así lo percibe, puede resultar más productivo en su desarrollo laboral, esto infiere que se deben adecuar los lineamientos internos de las empresas para mejorar la percepción de felicidad y de bienestar en los empleados, al mismo tiempo que se promueve la educación continua.

\section{REFERENCIAS BIBLIOGRÁFICAS}

Achor, S. (2010): “The Happiness Advantage”. New York: Random House Inc.

Brief, V. T. (2014): “Creating”, Veterinary Team Brief, 11.

Álvarez Nobell, A. y Muñiz Velázquez, J.A. (2013): "Felicidad y desarrollo de la cultura en las organizaciones, un enfoque psicosocial”, Revista Comunicación, 12, pp. 7-31.

Castellanos-Cereceda, R. (17 de Abril de 2013): “Marco internacional del bienestar subjetivo: debates, posturas e iniciativas”, Recuperado el 29 de 02 de 2016, de INEGI: http://www.inegi.org.mx/eventos/2013/Bienestar_subjetivo/doc/PRobertoCastellanos.pdf.

Chatenier, E., Verstegen, J., Biemans, H., Mulder, M. y Omta, O. (2010): "Identification of competencies for professionals in open innovation teams”, R\&D Management, 40(3), pp. 271-280. 
Climént Bonilla, J.B. (2012): "Redimensionando el significado y alcance de las competencias personales”, Actualidades Investigativas en Educación, 12(2), pp. 1-28.

Climént Bonilla, J.B. (2014): "Las competencias individuales: de las expectativas de terceros a la identidad personal”, Actualidades Investigativas en Educación, 9(2), pp. 1-20.

Coetzee, M., Bergh, Z. y Schreuder, D. (2010): “The influence of career orientations on subjective work experiences”, Journal of Human Resource Management, 8(1), pp. 113.

Essounga-Njan, Y. (2014): "Happiness and productivity: A cross-cultural review of the USA and France", Proceedings for The Northeast Region Decision Sciences Institute (NEDSI), pp. 833-840.

Field, L.K. y Buitendach, J.H. (2011): "Happiness, work engagement and organisational commitment of support staff at a tertiary education institution in South Africa", Journal of Industrial Psychology, 37(1), pp. 1-10.

Hudec, G., Susnea, I., Bihar, Ž. y Kirinić, V. (2015): “Teaching Creativity in Engineering”, Proceedings of the Multidisciplinary Academic Conference, pp. 1-5.

Johansen, V. (2014): "Entrepreneurship Education and Academic Performance", Scandinavian Journal of Educational Research, 58(3), pp. 300-314.

La Madriz, J. y Parra, J. (2016). “Competencias personales y profesionales aplicadas por gerentes bajo escenarios de incertidumbre económica”, Negotium, 11(33), pp. 69-98.

López, J. (2010). "La selección de personal basado en competencias y su relación con la eficiencia organizacional”, Perspectivas, 26, pp. 129-152.

Lyubomirsky, S. (2008): La ciencia de la felicidad: un método probado para conseguir el bienestar, Barcelona: Ediciones Urano.

Mahdavi Mazdeh, M., Razavi, S.M., Hesamamiri, R., Zahedi, M.R. y Elahi, B. (2013): “An empirical investigation of entrepreneurship intensity in Iranian state universities", Higher Education, 65(2), pp. 207-226.

Mantilla, M. y García, D. (2010): “Trabajo en equipos autodirigidos: competencias personales y conductas necesarias para su éxito”, Revista Venezolana de Gerencia, 15(49), pp. 51-71.

Moreno, Y. y Marrero, R. (2015): “Optimismo y autoestima como predictores de bienestar personal: Diferencias de género”, Revista Mexicana de Psicología, 32(1), pp. 27-39.

Marrero-Quevedo, R. J., Carballeira-Abella, M. y González-Villalobos, J. A. (2014): "Relación entre bienestar subjetivo, optimismo y variables sociodemográficas en estudiantes universitarios de la Universidad de San Luis Potosí en México", Universitas Psychologica, 13(3), pp. 1083-1098.

Nunnally, J C. (1967): Psychometric theory, New York: McGraw Hill.

OCDE (12 de 01 de 2016): OECD Better Life Index. Recuperado el 29 de 02 de 2016, de OECD Better Life Index: http://www.oecdbetterlifeindex.org/es/.

Olson, D. (2012): "Educating the Developing Mind: Towards an Overarching Paradigm”, Educational Psychology Review, 24(1), pp. 9-11.

Paschoal, T., Torres, C. y Barreiros, J. (2010): “Felicidad no Trabalho: Relações com Suporte Organizacional e Suporte Social”, ANPAD, pp. 1054-1072.

Posada, R. y Aguilar, O. (2012): "La felicidad como elemento del desarrollo sostenido desde la organización”, Revista del Centro de Investigación, 10(37), pp. 83-94. 
Ramírez, E. y Cárdenas, S. (2013): “Un análisis de la vinculación entre empresas mexicanas e instituciones de educación superior a partir de los resultados de la Encuesta Nacional de Vinculación”, Perfiles Educativos, 35(140), pp. 119-131.

Rey, B. (2014): “En torno a las palabras Competencia y Competencia Profesional”, Propuesta Educativa, 2(42), pp. 28-38.

Reynolds, C., Stevens, D. y West, E. (2013): “I'm in a Professional School! Why Are You Making Me Do This? A Cross-Disciplinary Study of the Use of Creative Classroom Projects on Student Learning”, College Teaching, 61 (2), pp. 51-59.

Ruiz Larraguivel, E. (2014): "Las empresas como espacios para el aprendizaje ocupacional, La experiencia educativa de los técnicos superiores universitarios”, Perfiles Educativos, 36(144), pp. 69-84.

Strand, T. (2011): "Metaphors of Creativity and Workplace Learning”, Scandinavian Journal of Educational Research, 55(4), pp. 341-355.

Straume, L. y Vittersø, J. (2015): "Well-Being at Work Some Differences Between Life Satisfaction and Personal Growth as Predictor of Subjective Health and Sick-Leave”, Journal of Happiness Studies, 16(1), pp.149-168.

Swaroop, P. (2016): "Effects of Happiness as a Brand Building Strategy”, International Journal of Management and Economics, 2(2), pp. 547-552.

Zak, P. y Winn, B. (2016): "Your Body at Work: Physiology, Neuroscience, and Leadership”, People \& Strategy, 39 (2), pp. 58-61. 


\section{ANEXO A: Cuestionario Aplicado ${ }^{1}$}

F1 En general, me considero una persona FELIZ

F2 $\quad$ En comparación con mis compañeros, me considero una persona FELIZ

Algunas personas son generalmente muy felices. Ellos disfrutan la vida sin importar lo que esté

F3 pasando, tratando siempre de obtener lo mejor de las cosas. ¿Qué tanto esta caracterización te describe?

Algunas nert

F4 Algunas personas no son muy felices o no parecen ser felices. ¿Qué tanto esta caracterización te describe?

B1 El bienestar depende de la percepción subjetiva que la persona tiene de su vida. Con respecto a esto, las condiciones de mi vida me hacen sentir totalmente realizado

\begin{tabular}{|c|c|c|}
\hline \multirow{4}{*}{ 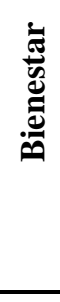 } & B2 & En la mayoría de las cosas que hago, mi vida está cerca de mi ideal. \\
\hline & B3 & Las actividades y ocupaciones que tengo me hacen sentir realizado \\
\hline & B4 & $\begin{array}{l}\text { Hasta ahora he conseguido las cosas que para mí son importantes en la vida }[1=\text { "En ningún } \\
\text { sentido" y } 7 \text { = "Totalmente”] }\end{array}$ \\
\hline & B5 & En gran medida, los éxitos y logros que tengo se los debo a mis amigos, compañeros y conocidos. \\
\hline & E1 & Los estudios que he realizado han llenado mis expectativas \\
\hline
\end{tabular}

E2 Considero que estoy realizando las actividades y estudios que más me agradan

E3 $\quad$ He logrado desarrollar mis conocimientos y considero que soy capaz de aprender por mi cuenta

E4 En mi universidad/trabajo existen actividades que me motivan a realizar cosas nuevas cada día

\begin{tabular}{l|l} 
E5 & El trabajo en equipo es la forma de trabajar que más prefiero.
\end{tabular}

E6 En algunas ocasiones he pensado en abandonar mis estudios

\begin{tabular}{l|l} 
C1 & Tengo definidos mis objetivos y mis metas por alcanzar en mi vida profesional y personal
\end{tabular}

C2 Considero que siempre logro mis objetivos y alcanzo mis metas

C3 $\quad$ Me gusta tener nuevos retos y los enfrento y resuelvo con actitud positiva

C4 Mis deseos de alcanzar mis objetivos han provocado una mejor manera de tomar decisiones

\begin{tabular}{|c|c|}
\hline C5 & Me considero más preparado y capacitado que mis compañeros \\
\hline C6 & El trabajo en equipo mejora mis conocimientos y me ayuda a cumplir mis objetivos. \\
\hline CO1 & En términos generales, ¿en qué medida se considera Ud. una persona competitiva? \\
\hline $\mathrm{CO} 2$ & $\begin{array}{l}\text { En términos generales, ¿̇en qué medida se considera Ud. una persona que acepta cuando se } \\
\text { equivoca? }\end{array}$ \\
\hline $\mathrm{CO} 3$ & $\begin{array}{l}\text { En términos generales, ¿en qué medida se considera Ud. una persona que asume las consecuencias } \\
\text { de sus errores? }\end{array}$ \\
\hline
\end{tabular}

CO4 En términos generales, ¿en qué medida se considera Ud. una persona emprendedora?

\footnotetext{
${ }^{1}$ Los reactivos en fondo gris fueron eliminados del estudio, no presentaron validez en el análisis de confiabilidad
} 